\title{
Los usos narrativos del incompletivo en algunas lenguas mayas
}

\section{The Narrative Uses of the Incompletive Marker in Some Mayan Languages}

\author{
Igor Vinogradov \\ Programa de Becas Posdoctorales en la UNAM, \\ Becario del Instituto de Investigaciones Antropológicas \\ email: happyjojik@yandex.ru \\ ORCID: orcid.org/0000-0003-4463-6468
}

Resumen:

Un discurso narrativo normalmente representa una secuencia de eventos pasados y terminados. En las lenguas mayas estas características aspectuales y temporales corresponden a la semántica de la categoría del completivo. Sin embargo, las formas verbales marcadas por el incompletivo también se encuentran en las narraciones en varias lenguas de la familia con mayor o menor frecuencia. En estas ocasiones el incompletivo desarrolla un significado secundario, muy parecido al del completivo. Se argumenta que los cambios de marcación de tiempo/aspecto aparecen por razones pragmáticas cuando el hablante enfatiza algún episodio dentro de la sucesión narrativa.

Palabras clave: discurso narrativo, tiempo/aspecto, lenguas mayas, completivo/incompletivo.

\section{Abstract:}

A narrative discourse normally represents a sequence of past and completed events. In Mayan languages, these aspectual and temporal characteristics correspond to the semantics of the incompletive category. However, verb forms marked with the incompletive are also found more or less frequently in narrations in several languages of the family. In these cases the incompletive develops a secondary meaning, very similar to that of the completive. It is argued that these changes in tense/aspect marking occur due to some pragmatic reasons, when the speaker emphasizes some episode within the narrative sequence.

Keywords: narrative discourse, tense/aspect, Mayan languages, completive/incompletive. 


\section{Introducción}

a estructura del discurso narrativo fue objeto de estudios lingüísticos de algunas lenguas de la familia maya. Por ejemplo, Hofling (1987) analiza las narraciones itza'; el trabajo de Maxwell (1987) se dedica al chuj; la tesis de Hull (2003) presenta una investigación del discurso narrativo en ch'orti', mientras que England (2009) describe los rasgos discursivos de las narraciones en mam. Asimismo, existen estudios discursivos sobre el maya clásico, la lengua de las inscripciones jeroglíficas; véase, por ejemplo, Josserand (1995) y Houston (1997). Los autores han señalado usos especiales que tienen algunos medios léxicos y gramaticales en un discurso narrativo y que normalmente no se encuentran en una conversación o en otros géneros de discurso. Comúnmente, se trata del uso de ciertas combinaciones fijas de palabras, algunas partículas discursivas y categorías verbales de tiempo, aspecto y modo en una lengua individual.

El presente trabajo se centra en los usos de la categoría del incompletivo en un discurso narrativo. ${ }^{1}$ Este tema particular se trata bajo un enfoque comparativo, considerando varias subdivisiones de la familia maya, tanto filogenéticas como areales. Sin embargo, este escrito no pretende alcanzar una muestra de lenguas que sea significativa estadísticamente, y por lo tanto no ofrece un análisis cuantitativo. Lo que se discutirá a continuación podría servir como un primer acercamiento al problema.

\section{El discurso narrativo}

Weinrich (1977) y Labov (1972) fueron de los primeros investigadores que, dedicándose al análisis del discurso, empezaron a estudiar la narrativa desde el punto de vista lingüístico. La narración se consideró como un género discursivo específico, junto con los demás géneros tales como argumentativo, descriptivo

\footnotetext{
${ }^{1}$ La versión preliminar de este estudio fue presentada en el congreso II Jornadas Internacionales y Transdisciplinarias: Espacios de diversidad e interculturalidad en el Sureste de México, Centroamérica y El Caribe, que se llevó a cabo los días 12, 13 y 14 de agosto de 2015 en San Cristóbal de las Casas (Chiapas). Agradezco a Telma Can Pixabaj por su cordial invitación a participar en dicho congreso. Igualmente, expreso mis agradecimientos al Programa de Becas Posdoctorales en la UNAM.
} 
o instructivo, por ejemplo. Labov (1972:359) define la narración como un método de recapitular experiencias pasadas ajustando una secuencia verbal de cláusulas a la secuencia de eventos ocurridos.

Fleischman (1991:78-79) introdujo la noción de la «norma narrativa» para determinar las características fundamentales de este particular género discursivo, atribuyéndole cuatro principios básicos. Primero, las narrativas hacen referencia a experiencias específicas que ocurrieron en un mundo pasado (real o imaginario). Segundo, el esqueleto de una narración se constituye por la secuencia de eventos, aunque el narrador puede añadir descripciones o comentarios, rompiendo el orden cronológico. El tercer principio dice que el orden de presentación no marcado en una narrativa es lineal en el tiempo. Las unidades narrativas (cláusulas) en el texto reflejan el orden en que se asume que ocurrieron los eventos en el mundo que se decribe. Y por último, una narración siempre transmite una manera particular de comunicar la información que puede reflejar la posición del narrador, sus emociones y sus relaciones con personajes o eventos descritos. Todo esto influye en su manera de narrar y, como se mostrará a continuación, afecta al uso de algunos medios verbales.

Cualquier narrativa tiene su estructura interna. Aparte de que la trama contiene distintos elementos, tales como introducción, complicación, clímax, desenlace, etc. (dependiendo del tipo particular de la narrativa), la narrativa no es simplemente una secuencia lineal de eventos ordenados cronológicamente, sino una configuración de eventos que tiene «textura» (Fleischman 1985:854). Es decir, algunos eventos o conjuntos de eventos (episodios) son más importantes para la trama que los otros (cf. Schiffrin 1981).

\section{El incompletivo dentro del sistema verbal en lenguas mayas}

Los sistemas verbales de diferentes lenguas del tronco mayense exhiben una variación notable; ello concierne tanto a la estructura morfológica y morfosintáctica en general, como al repertorio de categorías gramaticales y sus marcas respectivas (véase, por ejemplo, Schumann 1993). A pesar de esta gran diversidad, dos categorías - el completivo y el incompletivo — se encuentran dentro del sistema gramatical del verbo en todas las lenguas modernas de la familia (Vinogradov 2016). 
Los medios propiamente morfológicos de expresión de las categorías del completivo e incompletivo varían considerablemente según las lenguas individuales (véase el cuadro 1). Hay lenguas donde los marcadores del completivo e incompletivo preceden a la forma verbal, o sea, son prefijos, proclíticos o partículas prepositivas. Hay lenguas que marcan estas dos categorías exclusivamente por medio de sufijos. En fin, hay lenguas que exhiben el así llamado fenómeno de doble marcación. A este grupo pertenecen las lenguas mayas del subgrupo yucatecano y chol. En estas lenguas, al menos en cláusulas independientes, el completivo y el incompletivo se expresan por medio de un elemento auxiliar prepositivo que necesariamente está acompañado por un sufijo.

La lengua ch'orti' no comparte ninguno de los patrones mencionados arriba y por lo tanto representa una excepción. El ch'orti' no dispone de ningún morfema específico para expresar los significados del completivo ni del incompletivo. Esta distinción se mantiene solamente para los verbos intransitivos, y se indica junto con los significados de número y persona del único argumento. El ch'orti' tiene dos distintos juegos de marcadores personales que hacen referencia al argumento del verbo intransitivo. En el incompletivo se utilizan los marcadores personales prepositivos (el juego «C»), mientras que en el completivo se utiliza el juego pospositivo («B»); véase del Moral (1988) y Schumann (2007) para una descripción más detallada. El cuadro 1 resume esta variedad de expresiones morfológicas.

Cuadro 1. La marcación morfológica del incompletivo en algunas lenguas mayas.

\begin{tabular}{|c|c|c|c|}
\hline Tipo de marcación & Lengua & Marcadores & Ejemplos \\
\hline \multirow{2}{*}{ Marcación prepositiva } & chuj & $\mathrm{tz}=$ & $t z=w-i\left|=e x{ }^{\prime}\right| o s(2 p l) v. e o^{\prime}$ \\
\hline & tseltal & ya, ya $x-$ & ya $x$-lok' = on '(yo) salgo' \\
\hline \multirow{2}{*}{ Marcación sufijal } & huasteco & -al, -iil & $u=k a p-w-a l^{\prime}(y o) c o m o '$ \\
\hline & chontal & $-e^{\prime}$, -an, -än, -en & $k \ddot{a}=k^{\prime} u x-e^{\prime \prime}(y o) c o m o^{\prime}$ \\
\hline «Doble marcación» & $\begin{array}{l}\text { chol } \\
\text { yucateco }\end{array}$ & $\begin{array}{l}\text { mi, muk' }+-e l,-a \tilde{n} \\
k=+-i k,-a l,-e l\end{array}$ & $\begin{array}{l}\text { mi } \mathrm{k}=\mathrm{il}-\mathrm{an} \tilde{n}=\text { ety 'te veo' } \\
k=\text { in xok-ik '(yo) leo' }\end{array}$ \\
\hline Marcación cumulativa & ch'orti' & {$[« J$ uego $C »]$} & $i^{\prime}$-ixin '(tú) vas' vs. ixin = e't'fuiste' \\
\hline
\end{tabular}

Fuente: Los datos se derivan de Buenrostro (2010), Polian (2013), Kondic (2012), Knowles (1984), Vázquez (2011) y Bohnemeyer (2002). Los datos del ch'orti' provienen del trabajo de campo del autor.

Ahora, en cuanto al lugar que ocupan las categorías del completivo e incompletivo en el sistema verbal, cabe notar que no necesariamente son las únicas 
categorías que constituyen el sistema gramatical de tiempo/aspecto/modo. Dentro del mismo paradigma, junto con el completivo e incompletivo pueden encontrarse otros miembros, dependiendo de la lengua particular. Por ejemplo, chol también distingue el progresivo; en k'iche' existe el optativo, mientras que en mam el completivo se desintegra en el completivo reciente y el completivo remoto. Estas categorías «adicionales» ejercen influencia significativa en los significados del completivo e incompletivo (Vinogradov 2014b).

Aun reconociendo la variación semántica que se da entre las lenguas individuales, se puede generalizar, de una manera muy aproximada, que el completivo indica que la acción es concluida, mientras que el incompletivo se usa para las acciones no concluidas. En la mayoría de las gramáticas descriptivas estas dos categorías se definen referiéndose a la noción de la «terminación». Por ejemplo, en acateco «el completivo es el aspecto que hace referencia a las acciones que ya se terminaron, ya sea de forma inmediata o en un periodo de tiempo mayor», y «el incompletivo es el aspecto que hace referencia a las acciones que no están terminadas» (Zavala 1992:69-70). Sin embargo, no está claro qué es lo que se quiere decir con la idea de «terminación» y cuál es el momento de referencia para observar si la acción está terminada o no. De ahí surge el problema semántico de la delimitación entre el tiempo y el aspecto verbal que aparece cuando se trata de los significados del completivo e incompletivo (véase Vinogradov 2016).

Según las definiciones admitidas, por ejemplo, por Comrie (1976, 1985), el tiempo gramatical relaciona el momento de la situación con referencia a algún otro momento que a menudo (pero no necesariamente) coincide con el momento de la enunciación. Cuando el momento de referencia coincide con el momento de la enunciación, se trata del «tiempo absoluto»; en caso contrario, se suele hablar del «tiempo relativo». Por lo tanto, el tiempo tanto absoluto como relativo se distingue del aspecto gramatical que representa distintas maneras de ver la estructura interna de la situación. Es decir, el aspecto puede hacer referencia a una parte espécifica de una situación prolongada (como el aspecto inceptivo que realza la etapa incial de una acción o el aspecto prospectivo que realza el estado que precede inmediatamente a la acción) o puede hacer referencia a la manera de observar una situación completa (como el aspecto habitual que enfatiza la reiteración de una acción o el aspecto delimitativo que realza ambos límites temporales de una acción que tiene duración). Muy a menudo, en vez de poseer de un medio morfológico específico para cada significado individual, las lenguas agrupan los significados elementales semánticamente cercanos en conjuntos as- 
pectuales, los más comunes de los cuales son el «perfectivo» y el «imperfectivo» (véanse más detalles en Dahl 1985:69-89, Gvozdanovic 2012). Cabe notar que algunos autores pueden utilizar el término «aspecto» en un sentido un poco diferente, sobre todo anexando al aspecto los significados del tiempo relativo (véase Klein 1994), por ejemplo.

Sin entrar en detalles semánticos no relevantes para el problema a tratar, sería justo decir que una acción prototípica que se expresa por el completivo en las lenguas mayas es una acción pasada y perfectiva desde el punto de vista aspectual. Es decir, el completivo marca tanto acciones inherentemente instantáneas ('reventar', 'estornudar') u observadas como puntuales en su etapa final ('morirse', 'sacar el machete'), como también acciones que tienen una duración en el tiempo, pero explícitamente limitada ('dormir dos horas'). El incompletivo, siendo la parte opuesta al completivo, se define de manera negativa; se utiliza cuando una acción no representa un evento completado o concluido. Por ejemplo, el incompletivo puede hacer referencia a eventos pasados si aspectualmente son habituales o progresivos. Como ambas categorías involucran los dos dominios semánticos a la vez: temporal y aspectual, se denominan categorías de «tiempo/ aspecto».

\section{Los usos del incompletivo en una narración}

Como fue señalado anteriormente, un discurso narrativo normalmente representa una secuencia de eventos pasados y terminados. Estas características aspectuales y temporales corresponden perfectamente a la semántica del completivo en las lenguas mayas. La semántica del incompletivo que básicamente abarca las situaciones progresivas y habituales (Vinogradov 2014b) no encaja bien en un discurso narrativo, ya que las situaciones progresivas no son terminadas por definición y las situaciones habituales no pueden ser enfiladas sucesivamente debido a que no están localizadas fijamente en el eje temporal. Entonces, dentro de una narración no se espera encontrar formas verbales marcadas por el incompletivo, por lo menos si uno no toma en cuenta posibles comentarios u observaciones del narrador. No obstante, ello suele pasar en varias lenguas de la familia maya con mayor o menor frecuencia.

Antes de presentar algunos ejemplos de dicho fenómeno en diferentes lenguas hay que hacer una breve nota metodológica. Como en este apartado se 
utilizan las fuentes de datos secundarias, es posible que las traducciones propuestas reflejen interpretaciones del texto original por parte de los investigadores o hablantes nativos de las lenguas respectivas que colaboraron con ellos. Estas interpretaciones pueden afectar el uso de categorías de tiempo/aspecto, por ejemplo, ignorando el contexto o adaptándose a las directivas del idioma español; nótese que aun en español el uso de categorías gramaticales del verbo tiene sus peculiaridades muy significativas cuando se trata del género narrativo (cf. Silva-Corvalán 1983). Sin embargo, a continuación se consideran solamente los eventos que satisfacen los criterios de la «norma narrativa» (Fleishman 1991) discutidos arriba. Por lo tanto, se supone que todas las cláusulas hacen referencia a eventos concluidos en el pasado. Las traducciones que acompañan a los ejemplos sirven nada más para aclarar el significado y no pretenden reflejar correctamente las propiedades temporales o aspectuales de los eventos. ${ }^{2}$

(1) Tsotsil (Laughlin 1977:28, 59)

a. bweno, o la jun vinik icham la yajnil

$\begin{array}{llllll}\text { bweno } & \text { la jun vinik } & \text { i-cham la } & \text { y-ajnil } \\ \text { bueno EXIST } & \text { REP uno hombre } & \text { com-morir } & \text { REP } & \text { 3A-esposa }\end{array}$

'Había un hombre cuya mujer se murió'

$\begin{array}{lllllll}\text { batz'i } & \text { chok' }^{\prime} & \text { la } & \text { ta } & \text { jmek } & \text { ti } & \text { vinike } \\ \text { bats'i } & \text { ch-ok' } & \text { la } & \text { ta } & \text { jmek } & \text { ti } & \text { vinik }=e \\ \text { muy } & \text { INC-Horar } & \text { REP } & \text { PREP } & \text { mucho } & \text { DEF } & \text { hombre = ENCL }\end{array}$

'El hombre lloró mucho'

chk'ot la ta mukenal

$\begin{array}{llll}\text { ch- } k \text { 'ot la ta muk-enal } \\ \text { INC-llegar REP PREP } & \text { enterrar-LOC } \\ \text { 'Se fue al panteón' }\end{array}$

\footnotetext{
${ }^{2}$ La ortografía y la traducción de los fragmentos narrativos aparece tal como está en la fuente. Si la fuente ofrece una traducción al inglés, la versión aproximada en español es mía. Cuando la fuente no presenta glosas, las glosas son mías. Se utilizan las siguientes abreviaciones: A - juego A de marcadores de persona, AFE - afectivo, AGN - agente, APL - aplicativo, CAUS - causativo, COM - completivo, DEF - artículo definido, DEM - demostrativo, DER - sufijo de derivación, DIM - diminutivo, DIR - direccional, ENCL - enclítico, ENF - énfasis, EXH - exhortativo, EXIST - predicado existencial, HON - honorífico, INC incompletivo, INM - inmediato, LOC - locativo, NEG - negación, NMLZ - nominalización, PAC - paciente, PAS - voz pasiva, PD - pasado discontinuo, PL - plural, PREP - preposición, REL - relativizador, REP - reportativo, SD - sufijo direccional, SUBJ - subjuntivo. Los morfemas «cero» no se indican.
} 
chk'ot la ok'uk

$\begin{array}{lll}\text { ch-k'ot } & \text { la } & \text { ok'-uk } \\ \text { INC-llegar } & \text { REP } & \text { Horar-SUBJ }\end{array}$

'Llegó a llorar'

ital la jun vinik

i-tal la jun vinik

com-venir REP uno hombre

'Apareció (lit., «vino») un hombre'

b. ta la sbutan lok'el xchak ta ventana ti antze ta xxokon svayeb

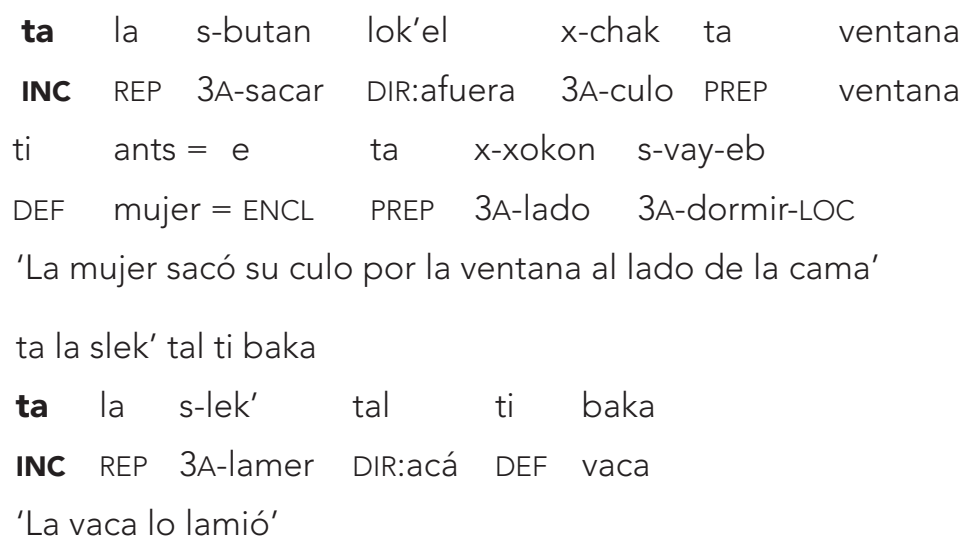

En (1) se presentan dos fragmentos de cuentos tsotsiles. Las cláusulas en el incompletivo en (1a) forman un pequeño episodio que está «encuadrado» en el marcaje completivo. El primer uso del incompletivo en (1a) podría ser explicado por la duración del evento 'llorar' que no puede suceder de manera instantánea debido al significado léxico del verbo; pero los demás eventos en (1a), tanto marcados por el completivo como por el incompletivo, son claramente pasados, completados y terminados al momento de narrar. El evento 'lamer' en (1b) también puede ser interpretado como una situación que tiene duración y por lo tanto como una situación aspectualmente imperfectiva. Sin embargo, la misma interpretación no se aplica al evento 'sacar' en la primera cláusula en (1b). Este evento por sí mismo no necesariamente es instantáneo; no obstante, lo que se realza en este contexto es su momento final, cuando se alcanza su fin inherente. 
(2) Chol (Whittaker \& Warkentin 1965: 124-125)

jini yujilbä ipejkäntel tsi' päyä tilel

$\begin{array}{llllll}\text { jiñi } & \text { y-ujil = bä } & \text { i-pejk-äñ-ty-el } & \text { tsi' } & \text { päy-ä } & \text { tyilel } \\ \text { DEM } & \text { 3A-saber }=\text { REL } & \text { 3A-hablar-DER-PAS-NMLZ } & \text { com.3A } & \text { Ilamar-COM } & \text { DIR:acá }\end{array}$

'Invitaron a la persona que sabe hablar [a los espíritus]'

che' jach, tsa' kaji ti k'ay, mi' poj mel

che' jach tsa' kaj-i tyi k'ay mi' poj mel

así sólo COM empezar-COM PREP canto INC.3A HON hacer.NMLZ

'Empezó a cantar, lo hacía un rato'

che' jini mi yujtel ti k'ay, ñäch'äl che' poj jalel

che' jiñi mi y-ujty-el tyi k'ay ñäch'äl che' poj jal-el

así DEM INC 3A-terminar-NMLZ PREP canto quieto así HON tardarse-NMLZ

'Entonces cuando terminó de cantar, permaneció tranquilo un rato'

che' jini mi' yäl, «wä'x an atat»

che' jiñi mi' $y$-äl wä' $=x \quad$ añ a-tyaty

así DEM INC.3A 3A-decir.NMLZ aquí =ya EXIST 2A-padre

'Entonces dijo, "Aquí está tu padre»'

che' mi' poj al jini yujilbä ipejkäntel

che' mi' poj al jiñi $\quad y$-ujil = bä i-pejk-äñ-ty-el

así INC.3A HON decir.NMLZ DEM 3A-saber = REL 3A-hablar-DER-PAS-NMLZ

'Así dijo el quien sabe hablar [a los espíritus]'

El fragmento (2) de una narración chol ilustra el cambio de marcación de tiempo/aspecto. Las dos primeras cláusulas aparecen en el completivo, mientras que todas las siguientes son marcadas por el incompletivo. En esta ocasión tampoco se encuentra explicación satisfactoria del uso del incompletivo que sea basada en las propiedades semánticas de los eventos descritos. Por ejemplo, los eventos 'empezar a cantar' (marcado por el completivo) y 'terminar de cantar' (marcado por el incompletivo) describen diferentes fases del mismo proceso. Pero ambas, la inicial y la final, representan situaciones puntuales en el pasado siendo momentos instantáneos en una escala temporal. Por lo tanto, ambos eventos semánticamente corresponden al completivo y no al incompletivo, y la diferencia de marcación queda sin explicación.

Es importante notar que fuera del contexto narrativo, las situaciones denotadas por los verbos que llevan marcadores del incompletivo no se interpretan por 
hablantes nativos como pasadas ni perfectivas. Al elicitar las formas verbales en el incompletivo presentadas en (2), la interpretación que se ofrece normalmente hace referencia a las acciones habituales o a las acciones que están en proceso.

(3) Uspanteco (Can Pixabaj 2006: 650-651)

sik' wunaq chi jnaqaj richoch xen jkorl man anm li

sik' wunaq chi j-naqaj r-ichoch $\mathbf{x}$-en j-korl man anm li

AFE hombre PREP 3A-cerca 3A-casa COM-hacer 3A-corral DEF mujer DEM

'Cerca de la casa del señor la señora hizo su corral'

toons tril qus tra íchij

toons t-r-il qus tra íchij

entonces INC-3A-ver bien DIM hierba

'Entonces vio bonito las hierbas'

ril sik wunaq

t-r-il sik wunaq

INC-3A-ver AFE hombre

'Vio el hombre'

tons tijb'ij sik' anm

tons $\quad \mathbf{i}$-j-b'ij sik' anm

entonces INC-3A-decir AFE mujer

'Entonces la pobre mujer le decía'

Una cláusula en el completivo inicia el fragmento (3), pero después el marcaje se cambia y las tres siguientes formas verbales están en el incompletivo, aunque desde el punto de vista temporal y aspectual, los eventos siguen siendo completados y pasados, y consituyen una secuencia cronológica: primero la señora construyó el corral, después el hombre vio las hierbas, y luego la señora le habló. La traducción de la última cláusula parece reflejar la forma uspanteca a la fuerza, y no suena naturalmente. Dado que la oración se encuentra dentro de una secuencia narrativa (véase la discusión arriba), es muy probable que se trate de un evento completado y no de una acción que suceda habitualmente o que esté en proceso en el momento de referencia. 


\section{Posibles propuestas de análisis}

En algunas lenguas mayas, el incompletivo desarrolla un significado secundario en las narraciones, muy parecido al significado básico que tiene el completivo fuera del discurso narrativo. Como los eventos narrativos pertenecen al pasado y representan acciones perfectivas desde la perspectiva aspectual (por ejemplo, puntuales o completadas), no requieren un medio gramatical que pueda expresar distinciones temporales o aspectuales, importantes para un discurso «ordinario» pero no para un discurso narrativo. Entonces, surge la pregunta, ¿qué es lo que condiciona la distribución entre el completivo y el incompletivo? Parece que se pueden proponer al menos tres hipótesis al respecto.

England (2009:217-218), al analizar los patrones del uso del incompletivo en las narraciones en mam, argumenta que el incompletivo no cambia su significado y sigue siendo utilizado como marcador del aspecto progresivo, es decir, cumple con una de sus funciones principales. Lo que pasa en una narración es que este significado no se aplica a la situación descrita por el verbo, sino a la propia situación de narrar. En otras palabras, el incompletivo en un discurso narrativo indica que la narración se está desarrollando poco a poco y todavía está en proceso. Esta propuesta concuerda con el uso prevalente del incompletivo en un discurso narrativo mam (4).

(4) Mam (England 2009: 217)

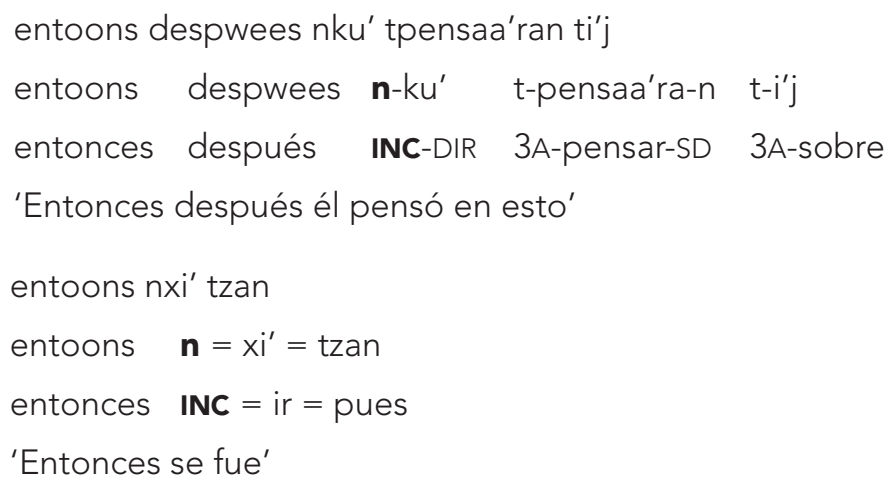


En el fragmento (4), el incompletivo funciona como una sustitución para el completivo y sin restricción alguna se usa en relación con eventos pasados, sin importar que sean instantáneos o prolongados, dependiendo del significado léxico del verbo particular. En estos ejemplos no se observan cambios de marcación; más bien, se trata del uso del incompletivo como la categoría «default» en un discurso narrativo. England (2009:218) identifica solamente cuatro ocasiones cuando se utilizan otras categorías de tiempo/aspecto: en introducciones y conclusiones de un cuento; en citas directas; en comentarios del narrador y cuando se reestructura el orden cronológico. Sin embargo, aunque hay todavía una considerable falta de datos, parece que este uso extremadamente frecuente del incompletivo en las narraciones en mam no se comparte en la mayoría de las demás lenguas mayas.

Otra idea que puede estar detrás de los usos del incompletivo en un discurso narrativo tiene que ver con el momento de referencia temporal, que para el caso, cuando se expresa el tiempo relativo, no necesariamente coincide con el momento de hablar. Es probable que cuando el incompletivo se utiliza para describir eventos pasados y completados que forman parte de la secuencia narrativa, el momento de referencia se desplace aunque este desplazamiento no se indique explícitamente. No corresponde con el momento actual en que sucede el acto de narrar, sino con el momento en que suceden las acciones descritas en la narración. Entonces, los eventos no se presentan como pasados y completados, ya que relativamente al momento de referencia no lo son, y para indicar que son simultáneos al momento de referencia se utiliza el incompletivo. Esta explicación funciona bien para los casos como el del mam (ej. 4), donde el uso del incompletivo prevalece significativamente al uso de otras categorías de tiempo/aspecto. Sin embargo, para los casos como el del tsotsil (ej. 1) o chol (ej. 2), no se explica por qué el momento de referencia en algunas ocasiones coincide con el momento de hablar (y se usa el completivo), pero en otras coincide con el momento cuando ocurren los hechos narrados (y se usa el incompletivo).

La tercera hipótesis, que parece ser más universal, tiene que ver con la pragmática, o sea, con diferentes modos de presentar la información. El cambio de marcaje del completivo al incompletivo y viceversa se debe a las relaciones subjetivas que existen entre el narrador y los eventos que forman la secuencia narrativa. En términos propuestos por Bertinetto (1987), se trata de «un tipo de uso metafórico» de categorías aspectuales. El cambio de marcaje es una herramienta que permite al narrador poner más énfasis en unos eventos y menos en otros, 
y de esta manera facilita la percepción de la narración por la parte del oyente. Varios ejemplos discutidos con anterioridad ilustran que las cláusulas marcadas por el completivo y las marcadas por el incompletivo a menudo se reúnen en grupos, y así van formando los episodios individuales, o sea, las partes integrantes de la narración. Es probable que los episodios y no las cláusulas aisladas sean unidades elementales que participan en este proceso de enfatizar. El cambio de marcación indica sus límites, donde termina un episodio y empieza otro. En (5) aparece un ejemplo donde el episodio de primer plano está marcado por el incompletivo.

(5) Tsotsil (Laughlin 1977: 51)

mu to ox la sna', mi ja' smalal

mu to ox la s-na' mi ja' s-malal

NEG aún PD REP 3A-saber si ENF 3A-marido

'Ella aun no sabía que [el zopilote] era su marido'

ta la smaj ech'el

$\begin{array}{llll}\text { ta } & \text { la } & \text { s-maj } & \text { ech'el } \\ \text { INC } & \text { REP } & \text { 3A-golpear } & \text { DIR:afuera }\end{array}$

'Lo golpeó'

ta la sk'asbe yakan ta te'

ta la s-k'as-be y-akan ta te'

INC REP 3A-romper-APL 3A-pierna PREP palo

'Le rompió las piernas con un palo'

ta la xjatav ta jol na

ta la X-jatav ta jol na

INC REP INC-escapar PREP cabeza casa

'[Él] escapó al techo de la casa'

yo'onuk la ochuk ta yut na pero mu xa k'u xcha'le

y-o'on-uk la och-uk ta y-ut na

3A-corazón-SUBJ REP entrar-SUBJ PREP 3A-adentro casa

pero mu xa k'u $x$-cha'le

pero NEG ya qué INC-actuar

'Quiso entrar en la casa pero ya no podía hacer nada' 
El fragmento (5) es retomado de la parte final del cuento, y describe el clímax. La mujer, sin saber que su marido se había convertido en zopilote, lo está matando a golpes. Otro ejemplo similar viene del chontal (6).

(6) Chontal (Pérez González 2006:50-55)

a. i ke u pase noj gran na' piyo'

i ke u = pas-e noj gran na' piyo'

y que $3 A=$ salir-INC grande grande hembra pollo

'Y que va saliendo la gallinota'

entonse ni ajkoyote $u k^{\prime} u x i$

entonse ni aj-koyote $u=k^{\prime} u x-i$

entonces DEF AGN-coyote $3 \mathrm{~A}=$ comer-COM

'Entonces el coyote se la comió'

b. i ke u yä'ben ni lugar kada ya an ni ajkoyote

i ke uy $=\ddot{a}$-b-en ni lugar $k a=d a$ ya an ni aj-koyote

y que $3 A=$ dar-APL-INC DEF lugar que = DEM ya EXIST DEF AGN-coyote

'Y que le enseña el lugar donde estaba el malvado coyote.'

i ke u tsämsen ni ajsakya

i ke $u=$ tsäm-s-en ni aj-sak-ya

y que $3 A=$ matar-CAUS-INC DEF AGN-buscar-NMLZ

'Y que lo mata el cazador'

entonse ni ajt'ul u yälbi, «kola por ni ixim»

entonse ni aj-t'ul uy =äl-b-i kola por ni ixim

entonces DEF AGN-conejo 3A=decir-APL-COM EXH por DEF maíz

'Entonces el conejo le dijo, «Vamos por el maíz»'

c. entonse ajsakya a t'äbi

entonse aj-sak-ya a t'äb-i

entonces AGN-buscar-NMLZ INM subir-COM

'Entonces el cazador se subió'

i tänxin ni k'ajte', ke u xulkan ni te'ba por k'un

i tänxin ni k'ajte' ke $u=x u l-k-a n \quad n i$

y en.medio DEF puente que $3 A=$ quebrar-PAS-INC $D E F$

$t^{\prime}=b a \quad$ por $k^{\prime} u n$

palo $=E N C L$ por podrido

'Y, a la mitad del puente, que se quiebra el palo podrido' 
Todos los ejemplos (6) son de la misma narración que generalmente está en el completivo, pero algunas cláusulas aparecen en el incompletivo. Se observa que el traductor logró encontrar un medio específico para transmitir el uso del incompletivo en la versión original de chontal a español. Es la construcción «y que» más la forma verbal en el tiempo presente. Dado que son eventos de la secuencia narrativa, la traducción más «natural» sería por medio de las formas en el pretérito: «salió» (6a), «enseñó» y «mató» (6b) y «se quebró» (6c). Probablemente, las cláusulas que reciben la marcación incompletiva son las que describen sucesos más importantes para el desarrollo de la trama. Son los puntos culminantes de las partes respectivas del cuento, y el incompletivo sirve para destacarlos de otros eventos o episodios menos importantes.

Como fue ilustrado anteriormente, ambas categorías, el completivo y el incompletivo, pueden marcar tanto episodios de fondo como de primer plano, según la estrategia que aplica la lengua particular; compárense, por ejemplo, los fragmentos (1a) y (5) en tsotsil. Una solución teórica posible de esta ambigüedad es suponer que lo que se marca no son los episodios como tales, sino los confines entre ellos. Y entonces, los medios verbales de marcación no son las categorías del completivo e incompletivo por sí mismos, sino su alternancia (véase, por ejemplo, Wolfson 1979 y Vinogradov 2014a). El cambio de marcaje denota el confín entre dos episodios de distintos niveles de importancia. El completivo y el incompletivo obtienen su significado pragmático y pueden cumplir con sus funciones discursivas gracias a que no se quedan aislados dentro del sistema gramatical del verbo, sino están opuestos uno a otro.

\section{En lugar de conclusiones}

Cabe notar que se observa un paralelo funcional entre el uso de categorías verbales en una narración maya y el empleo del así llamado «tiempo presente histórico» en varias lenguas europeas (ej. 7-8), incluyendo el antiguo griego y el latín.

(7) Español

a. En 1492 Cristóbal Colón llega a América.

b. En 1936 comienza la Guerra Civil en España. 
(8) Inglés (Schiffrin 1981:48)
a. And all of a sudden the buzzer sounds.
b. And all these guys [...] come [...] out.

Esta observación no es nueva; por ejemplo, Houston (1997) ofrece la misma analogía considerando los datos del maya clásico. Aunque en las lenguas mayas no se trata exclusivamente del tiempo, sino más bien de una combinación del tiempo y el aspecto verbal, la parte esencial del fenómeno es muy similar. En una narración, las distinciones temporales y aspectuales entre eventos se borran, y los medios que se suelen utilizar para ellas en un discurso conversativo, en un discurso narrativo, encuentran otra aplicación. Se utilizan para indicar relaciones entre distintos componentes de la narración y para expresar la evaluación subjetiva de la importancia de estos componentes por parte del narrador. En un discurso narrativo, las funciones semánticas del tiempo presente en las lenguas europeas se convierten en funciones pragmáticas; y el mismo cambio aparentemente ocurre con el aspecto incompletivo en algunas lenguas mayas.

Esta nota presentó las evidencias de que el fenómeno de emplear el incompletivo en el sentido del completivo bajo algunas circunstancias pragmáticas existe en la familia maya y no está restringido por alguna lengua particular. Se discutieron tres hipótesis al respecto:

- el incompletivo en un discurso narrativo indica el aspecto progresivo, pero no lo aplica a la situación descrita por el predicado, sino metafóricamente al proceso de narrar;

- el incompletivo indica el traslado del momento de referencia temporal desde el momento de narrar al momento en que suceden los eventos narrados;

- el cambio de marcación permite al narrador poner más énfasis en unos episodios de la secuencia narrativa y menos en otros.

Solamente fueron considerados algunos ejemplos de cinco lenguas que pertenecen a tres distintas ramas de las seis que existen dentro de la familia maya. La investigación futura podrá generalizar el problema ampliándose a las demás lenguas y a los demás subgrupos de la familia y profundizando el análisis de condiciones semánticas y pragmáticas bajo las cuales sucede dicho cambio de marcación de tiempo/aspecto. De mucho interés sería un estudio cuantitativo que permita obtener información acerca de las frecuencias del uso de distintas categorías verbales en distintas funciones dentro del discurso narrativo. 
Aunque hoy en día se observan grandes éxitos en cuanto a la tarea de documentación y descripción lingüística de las lenguas indígenas de Mesoamérica y de las lenguas mayas en particular —véase, por ejemplo, el breve resumen en Zavala (2010)_, el discurso sigue siendo el nivel de lenguaje que recibe la menor atención científica cuando se trata de las lenguas poco estudiadas. La continuación y extensión de la presente investigación podría contribuir al desarrollo de estudios discursivos sobre las lenguas indígenas habladas en Mesoamérica y en el mundo en general.

\section{Fuentes de consulta}

Bertinetto, Pier Marco, 1987, "Structure and origin of the narrative imperfect», en Anna Giacalone-Ramat, Onofrio Carruba y Giuliano Bernini (eds.), Papers from the $7^{\text {th }}$ International Conference on Historical Linguistics, Ámsterdam y Filadelfia, John Benjamins, pp. 71-85.

Bohnemeyer, Jürgen, 2002, The grammar of time reference in Yukatek Maya, Múnich, Lincom.

Buenrostro, Cristina, 2010, «Estructura de la palabra verbal en chuj de San Mateo Ixtatán», Lingüística Mexicana, 5(2), pp. 193-216.

Can Pixabaj, Telma (coord.), 2006, Gramática descriptiva Uspanteka, Guatemala, OKMA.

Comrie, Bernard, 1976, Aspect, Cambridge, Cambridge University Press.

— 1985, Tense, Cambridge, Cambridge University Press.

Dahl, Östen, 1985, Tense and aspect systems, Nueva York, Basil Blackwell.

England, Nora C., 2009, «To tell a tale: The structure of narrated stories in Mam, a Mayan language», International Journal of American Linguistics, 75(2), pp. 207-231.

Fleischman, Suzanne, 1985, «Discourse functions of tense-aspect oppositions in narrative: Toward a theory of grounding», Linguistics, 23(6), pp. 851-882.

— 1991, "Toward a theory of tense-aspect in narrative discourse», en Jadranka Gvozdanovic y Theo Janssen (eds.), The function of tense in texts, Ámsterdam, North Holland, pp. 75-97.

Gvozdanovic, Jadranka, 2012, "Perfective and imperfective aspect», en Robert I. Binnick (ed.), The handbook of tense and aspect, Oxford, Oxford University Press, pp. 781-802.

Hofling, Charles A., 1987, «Discourse framing in Itzá Maya narrative», Anthropological Linguistics, 29(4), pp. 478-488.

Houston, Stephen, 1997, «The shifting now: Aspect, deixis, and narrative in Classic Maya texts», American Anthropologist, 99(2), pp. 291-305.

Hull, Kerry M., 2003, "Verbal art and performance in Ch'orti' and Maya hieroglyphic writing», tesis de doctorado, Austin, Universidad de Texas. 
Josserand, Kathryn J., 1995, «Participant tracking in Maya hieroglyphic texts: Who was that masked man?», Journal of Linguistic Anthropology, 5(1), pp. 65-89.

Klein, Wolfgang, 1994, Time in language, Londres y Nueva York, Routledge.

Knowles, Susan M., 1984, "A descriptive grammar of Chontal Maya (San Carlos dialect)», tesis de doctorado, Nueva Orleáns, Universidad Tulane.

Kondic, Snježana, 2012, «A grammar of South Eastern Huastec, a Mayan language from Mexico», tesis de doctorado, Universidad de Sydney/Universidad 2 Lumière de Lyon.

Labov, William, 1972, "The transformation of experience in narrative syntax», en William Labov (ed.), Language in the Inner City, Filadelfia, University of Pennsylvania Press, pp. 354-396.

Laughlin, Robert M., 1977, Of cabbages and kings: Tales from Zinacantán, Washington, Smithsonian Institution Press.

Maxwell, Judith, 1987, "Some aspects of Chuj discourse», Anthropological Linguistics, 29(4), pp. 489-506.

Moral, Raúl del, 1988, «Introducción al sistema verbal del chortí de Guatemala», Estudios de Cultura Maya, 17, pp. 397-421.

Pérez González, Benjamín, 2006, Los duendes del viento: Literatura chontal de Tabasco, Villahermosa, Gobierno del Estado de Tabasco.

Polian, Gilles, 2013, Gramática del tseltal de Oxchuc, México, CIESAS.

Schiffrin, Deborah, 1981, "Tense variation in narrative», Language, 57(1), pp. 45-62.

Schumann Gálvez, Otto, 1993, «Aspectos generales del sistema verbal en las lenguas mayas», en María Josefa Iglesias Ponce de León y Francesc Ligorred Perramon (eds.), Perspectivas antropológicas en el mundo maya, Madrid, Sociedad Española de Estudios Mayas, pp. 443-456.

- 2007, Introducción a la morfología verbal del chortí, Graz, Academic Publishers.

Silva-Corvalán, Carmen, 1983, «Tense and aspect in oral Spanish narrative: Context and meaning», Language, 59(4), pp. 760-780.

Vázquez Álvarez, Juan Jesús, 2011, "A grammar of Chol, a Mayan language», tesis de doctorado, Austin, Universidad de Texas.

Vinogradov, Igor, 2014(a), «Aspect switching in Tzotzil (Mayan) narratives», Oklahoma Working Papers in Indigenous Languages, 1, pp. 39-54.

_ 2014(b), «La categoría de incompletivo en las lenguas mayas: Un estudio comparativo de variación semántica», Studii de Gramatica Contrastiva, 21, pp. 65-82.

__ 2016, «Acerca de la semántica del completivo/incompletivo en las lenguas mayas», Cuadernos de Lingüística, 3(1), pp. 5-44.

Weinrich, Harald, 1977 [1964], Tempus: besprochene und erzählte Welt, Stuttgart, Kohlhammer.

Whittaker, Arabelle y Viola Warkentin, 1965, Chol texts on the supernatural, Oklahoma, Instituto Lingüístico de Verano.

Wolfson, Nessa, 1979, «The conversational historical present alternation», Language, 55(1), pp. 168-182.

Zavala Maldonado, Roberto, 1992, El Kanjobal de San Miguel Acatán, México, UNAM. 
Zavala Maldonado, Roberto, 2010, «El estado de la lingüística en Chiapas y Guatemala», en Gabriel Ascencio Franco (ed.), La antropología en Centroamérica: reflexiones y perspectivas, Tuxtla Gutiérrez, Red Centroamericana de Antropología, pp. 149-183. 